\title{
Neuraxial Opioid Analgesia
}

National Cancer Institute

\section{Source}

National Cancer Institute. Neuraxial Opioid Analgesia. NCI Thesaurus. Code C21073.

Analgesic delivery into the spinal axis. 